\title{
Normas y exigencias familiares con adolescentes que presentan problemas conductuales en UDIPSAI. Septiembre 2017- Enero 2018
}

\section{Norms and family demands with adolescents who present behavioral problems in UDIPSAI. September 2017- January 2018}

\author{
Paola Gabriela Ordoñez Santander ${ }^{1 *}$ y Sandra Maribel Barros Bernal ${ }^{1}$ \\ ${ }^{1}$ Universidad Católica de Cuenca \\ *paorsan89@hotmail.com
}

DOI: https://doi.org/10.26871/killkana_salud.v2i2.261

\begin{abstract}
Resumen
Objetivo: mostrar los resultados de normas y exigencias familiares utilizadas con adolescentes que presentan problemas conductuales en la Unidad de Diagnóstico e Investigación Psicopedagógica y de Apoyo a la Inclusión (UDIPSAI) que han sido diagnosticados con anterioridad y que no presentan sintomatología que pueda caracterizar un trastorno especifico. Materiales y Métodos: Para llevar a cabo esta investigación se utilizó la metodología cuali- cuantitativa, la muestra es de 50 padres o representantes y 50 hijos adolescentes ya que la Escala de Normas y Exigencias está destinada tanto para padres como para hijos; dicha escala pretende evaluar los factores inductivo, rígido e indulgente de los estilos de crianza en el sistema familiar. Resultados: la percepción de los jóvenes sobre normas y exigencias familiares corresponde al factor inductivo, seguido por el factor rígido y finalmente el factor indulgente. Conclusiones: realizar talleres en las instituciones educativas y centros vinculados a la atención integral de familias.
\end{abstract}

Palabras clave: autoritario, democrático, estilos de crianza, exigencias, normas, permisivo.

\begin{abstract}
Objective: to show the results of family norms and requirements used with adolescents who present behavioral problems in the Unit of Diagnosis and Research Psychopedagogical and Support for Inclusion (UDIPSAI) who have been previously diagnosed and who do not present symptoms that may characterize a disorder specific. Materials and Methods: To carry out this research, the qualitative-quantitative methodology was used, the sample is of 50 parents or representatives and 50 adolescent children since the Scale of Norms and Requirements is intended for both parents and children; This scale aims to evaluate the inductive, rigid and indulgent factors of parenting styles in the family system. Results: the perception of young people about family norms and requirements corresponds to the inductive factor, followed by the rigid factor and finally the indulgent factor. Conclusions: conduct workshops in educational institutions and centers related to comprehensive family care.
\end{abstract}

Key words: authority, democratic, parenting styles, requirements, rules, permissive.

\section{Introducción}

En los modelos bidireccionales según menciona López et.al ${ }^{1}$ se considera que existe una influencia mutua entre padres e hijos, es decir, tanto la conducta del adolescente como su desarrollo socioemocional estarían influidos por las prácticas de crianza, entendidas como comportamientos observables de los padres desde la primera infancia. Pero a su vez, el propio comportamiento del niño, así como otra serie de factores característicos del mismo, estarían modulando progresivamente este tipo de prácticas parentales.
Numerosos estudios han tratado de relacionar la conducta agresiva no sólo con los distintos estilos de crianza, sino también con otros constructos más concretos referentes a la interacción diaria con los hijos. Uno de estos es el establecimiento de límites, también denominado disciplina en algunas ocasiones, que hace referencia a la consistencia de las normas establecidas por los padres y la inducción razonada hacia su cumplimiento por parte de los hijos. ${ }^{2}$

Es necesario asegurar como menciona Torío et.al. ${ }^{3}$ que la mayor parte de padres no tienen un estilo de crianza específico o bien presentan pautas de crianza contradictorias. Además como los padres utilicen cada uno de los 
estilos no será sin repercusiones, al contrario, los diversos modelos tendrán consecuencias en la conducta y adaptación prosocial y emocional del niño. ${ }^{4}$

De esta forma según las entrevistas y valoraciones realizadas en UDIPSAI se han observado múltiples casos de adolescentes que presentan problemas conductuales, por lo que es pertinente investigar las normas y exigencias familiares para conocer los estilos de crianza desde una perspectiva tanto de padres o representantes como de los usuarios y definir si existe influencia de dichos estilos en el comportamiento.

Es por ello que el presente artículo hace referencia a las normas y exigencias familiares de adolescentes que presentan problemas conductuales, pretende analizar la percepción tanto de adolescentes hijos como de padres o representantes acerca de los factores inductivo, rígido e indulgente utilizados como método de crianza en el hogar.

En esta investigación al hablar de normas y exigencias se hace referencia a los límites y reglas que se mantienen en el hogar con los adolescentes que presentan problemas conductuales, en especial los que se muestran agresivos, irritables, presentan conductas oposicionistas por lo que es importante conocer acerca de las exigencias que se mantienen dentro del sistema familiar.

\section{Marco Teórico}

Valenzuela et.al. ${ }^{5}$ define a la adolescencia como "el período de vida comprendido entre los 10 y 19 años, durante esta etapa el adolescente se transforma en un individuo maduro en sus dimensiones física, sexual, psicológica y social y establece su propia identidad". Como se menciona anteriormente la adolescencia es una época de cambios en el proceso de transformación del niño o la niña en adulto, que se requieren enfrentar, de igual forma que se adquieren nuevas capacidades. ${ }^{6}$ El adolescente vive esta etapa de manera activa y no simplemente se experimenta de forma pasiva. $^{7}$

Si esta etapa no se lleva satisfactoriamente puede generar problemas afectivos y de comportamiento en la vida adulta, es por ello que se le debe otorgar la debida importancia. ${ }^{8}$ Según menciona Martínez et.al ${ }^{9}$ "el consumo de sustancias, los comportamientos delictivos y la conducta escolar disruptiva se presentan con mayor frecuencia en la adolescencia”.

Es por ello que es importante para el funcionamiento de una familia el establecimiento de límites y reglas, estos deben ser claros y definirse de la manera más precisa ya que los límites demarcan el espacio entre los miembros de un grupo familiar. ${ }^{10}$ La aplicación de normas, reglas, límites y exigencias son considerados importantes factores de protección y su aplicación se puede apoyar en principios de la psicología conductual del condicionamiento operante. ${ }^{11}$ De esta forma, según menciona Ramírez ${ }^{12}$ los estilos de socialización en la adolescencia se vinculan con la intensidad de la comunicación en las relaciones padreshijos.

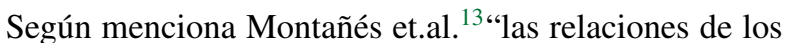
padres con los adolescentes y la existencia o no de conflictos, están muy condicionadas, por los estilos educativos empleados dentro del contexto familiar".

Es así que Becker ${ }^{10}$ distingue entre métodos disciplinarios que tienen el amor hacia el adolescente como instrumento principal y métodos autoritarios que son reacciones y comportamientos agresivos, de esta forma se tiene en cuenta disciplina y afecto como las dos variables que distinguen a unos padres de otros.

De igual forma Baumrind 14 "clasifica los estilos educativos como: autoritarios, democráticos y permisivos". Los padres autoritarios valoran la obediencia y utilizan medidas de castigo, los padres permisivos dotan al adolescente de autonomía, los libera de todo control y evitan usar la fuerza, finalmente los padres autoritativos o democráticos dirigen la autoridad utilizando el razonamiento y la negociación. ${ }^{15}$

Por lo tanto con estos estilos educativos existe un contraste de los extremos del autoritarismo y del permisivismo que se percibe como un defecto de la práctica parental que consiste en no compaginar la exigencia, estableciendo normas razonables de comportamiento con el cuidado cálido de las personas. ${ }^{16}$

Sin embargo los padres, madres o personas que están a cargo del cuidado de los adolescentes no se comportan en base a un solo estilo educativo, sino que utilizan varios estilos dependiendo de las situaciones. En definitiva, los estilos educativos, son los métodos o estrategias que se utilizan para influir en el comportamiento de los hijos e hijas, de esta forma se corrigen y se orienta su educación; además los estilos educativos incluyen habilidades como el aprendizaje de la comunicación, la imitación y el desarrollo de diferentes habilidades. ${ }^{17}$

Ramírez ${ }^{18}$ plantea que las prácticas de crianza que utilizan los padres se relacionan con los problemas conductuales en los hijos, por ejemplo afecto negativo, castigos físicos, control autoritario. Específicamente el afecto negativo se manifiesta con conducta agresiva, problemas de atención y problemas de conducta. Por lo tanto los problemas comportamentales se acrecientan en medida en que aumentan las prácticas de crianza inadecuadas.

\section{Metodología}

La investigación realizada es un estudio de campo que se llevó a cabo en la Unidad de Diagnóstico, Investigación Psicopedagógica y de Apoyo a la Inclusión UDIPSAI, participaron 50 adolescentes entre 12 y 19 años que presentan problemas de conducta y los correspondientes padres o representantes en el período de octubre y noviembre de 2017. La información se obtuvo mediante la Escala de Normas y Exigencias que está destinada a padres e hijos adolescentes, se aplicó de manera individual, por una parte a cada uno de los padres o representantes y por otro lado a los hijos.

El estudio tiene la característica de ser cuali-cuantitativo ya que nos otorga resultados medibles y comprobables y 
permite hacer una relación de la percepción de padres e hijos. De igual forma para la obtención de resultados se utilizó el programa informático SPSS.

\subsection{Instrumento}

Esta Escala tiene como objetivo identificar las normas y reglas o estilos educativos que utilizan las figuras parentales, lo cual se demuestra en el resultado de tres factores que son: inductivo, rígido e indulgente. ${ }^{19}$

Consta de 30 ítems a ser evaluados y se conforma por una Escala de normas y exigencias para padres ENE-P y una escala para hijos ENE-H; el reactivo contiene una escala de Likert que se compone de cinco respuestas y se puntúan de la siguiente forma: $1=$ nunca; 2 = pocas veces; $3=$ algunas veces; $4=$ a menudo; $5=$ siempre. En cuanto al alpha de Cronbach se tienen coeficientes de 0,85 , lo que indica un buen comportamiento psicométrico.

\subsection{Procedimiento}

Luego de obtener el consentimiento informado de padres $\mathrm{y}$ representantes de adolescentes que presentan problemas conductuales y acuden a UDIPSAI, se aplicó el instrumento de forma individual, en primer lugar a los adolescentes y posteriormente a padres o representantes, esto se realizó bajo observación y supervisión del examinador.

\section{Resultados}

Los resultados de la investigación serán expuestos a continuación en un análisis descriptivo:

El gráfico número 1 describe los resultados de la percepción que tienen los adolescentes acerca de las normas y reglas que utilizan sus padres en donde se puede observar que de los 50 adolescentes que participaron en la investigación 26 que corresponde al $52 \%$ responden a la forma inductiva, 22 que conforman el $44 \%$ responden al factor rígido y 2 que suman el $4 \%$ responden al factor indulgente. Estos resultados indican que mayoritariamente la percepción de los jóvenes sobre normas y exigencias familiares corresponde al factor inductivo, seguido por el factor rígido y finalmente el factor indulgente.

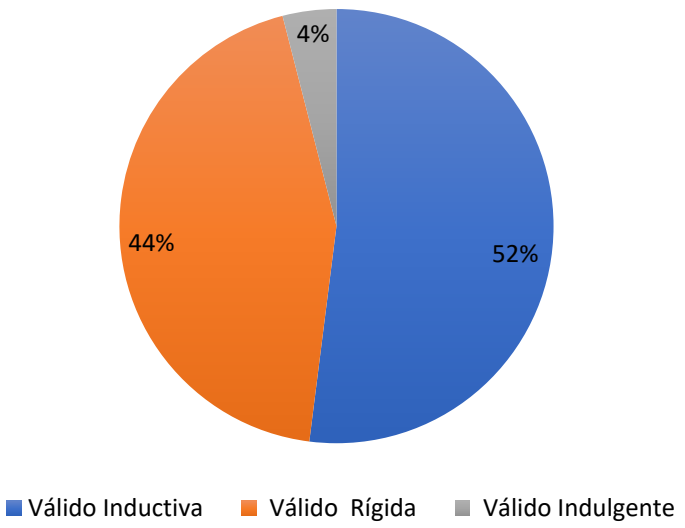

Fig. 1. Percepción acerca de normas y reglas de adolescentes- hijos con problemas conductuales en UDIPSAI.
De igual forma en el gráfico número 2 se pueden apreciar los resultados de la percepción de los padres o representantes en cuanto a normas y reglas. De los 50 padres que participaron en la investigación 29 que corresponde al $58 \%$ responden a la forma inductiva, 20 que conforman el $40 \%$ responden al factor rígido y 1 que implica el $2 \%$ responden al factor indulgente. Estos resultados indican que mayoritariamente la percepción de los padres sobre normas y exigencias familiares se inclina al factor inductivo, seguido por el factor rígido y finalmente el factor indulgente.

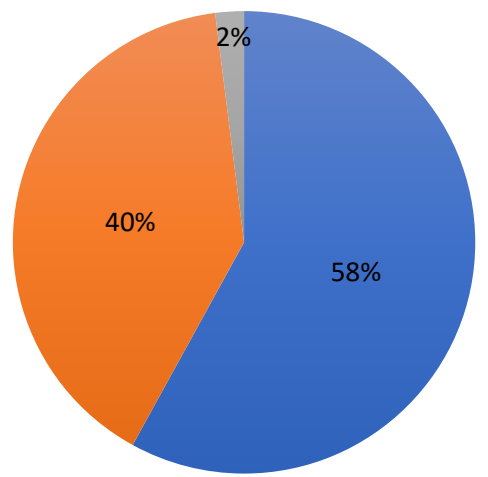

- Válidos Inductiva Válidos Rígida Válidos Indulgente

Fig. 2. Percepción de padres acerca de normas y reglas utilizadas con hijos adolescentes que presentan problemas conductuales en UDIPSAI.

Por otro lado en el gráfico número 3 se describe la relación que tiene la percepción de los padres con los hijos; se puede observar que de los 100 participantes de la investigación $26 \%$ de adolescentes y $29 \%$ de padres responden al factor inductivo, $22 \%$ de adolescentes y $20 \%$ padres se inclinan hacia el factor rígido, finalmente $2 \%$ de adolescentes y $1 \%$ de padres responden al factor indulgente. Estos resultados indican que existe relación en la percepción de padres e hijos con respecto a las normas y exigencias utilizadas en los estilos de crianza, se puede observar que padres y adolescentes están de acuerdo con que mayoritariamente se utiliza el estilo inductivo, seguido por el estilo rígido y el estilo indulgente con menor porcentaje.

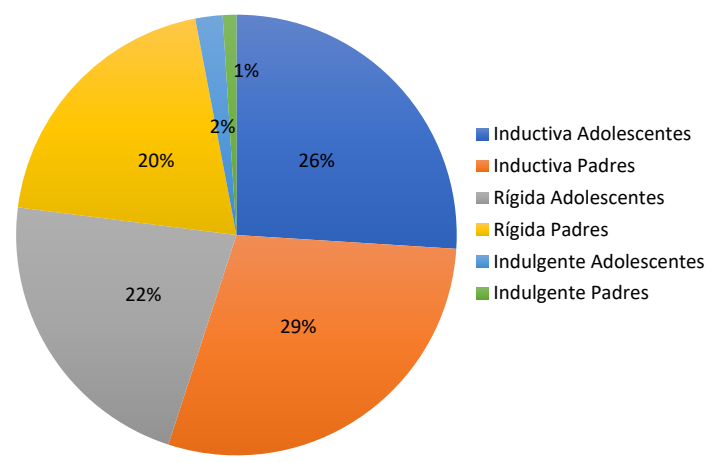

Fig. 3. Relación de la percepción de padres y adolescentes acerca de las normas y exigencias familiares. 


\section{Conclusiones y Recomendaciones}

En cumplimiento con el primer objetivo la percepción de los hijos adolescentes que presentan problemas conductuales en cuanto se refiere a las normas y exigencias impuestas por sus padres o representantes, demuestra que el sistema familiar se basa en el factor inductivo como método de crianza principal con un porcentaje de $52 \%$, en segundo lugar encontramos el factor rígido con $44 \%$ y finalmente el factor indulgente con un porcentaje de $4 \%$. Por lo tanto los resultados demuestran que un estilo de crianza basado en la autonomía y democracia puede influir en problemas conductuales en adolescentes, sin embargo se debe tener en cuenta que los resultados están expuestos a errores propios de todo instrumento, escala o cuestionario de evaluación.

En cuanto se refiere al segundo objetivo sobre la percepción de los padres acerca de normas y exigencias se puede evidenciar que al igual que los hijos los resultados dan a conocer que el factor inductivo con un $58 \%$ es el que obtiene los porcentajes más altos, seguido por el factor rígido con $40 \%$ y el factor indulgente con $2 \%$ es por este motivo que según los datos del estudio los padres cuentan con normas y exigencias en donde existe respeto y conocimiento mutuo.

Se hace referencia también a la relación que existe entre la percepción de padres e hijos y satisfactoriamente estos datos coinciden, otorgando al factor inductivo un porcentaje significativo, por lo tanto los resultados arrojados demuestran que el punto de vista de padres e hijos es acerca de un estilo de crianza con normas y exigencias que están orientadas a un sistema democrático, de autonomía, respeto y conocimiento mutuo; de igual forma los resultados obtenidos del estilo rígido no se alejan con un gran porcentaje del factor inductivo, por lo que muchos padres utilizan también con sus hijos normas y reglas en base a este factor; por otro lado el estilo indulgente obtuvo valores mínimos y se demuestra de esta forma que los estilos educativos basados en este factor son menores en la muestra analizada.

Una vez obtenidos estos datos se hace una comparación con el estudio de Torio ${ }^{3}$ en donde se evalúan los estilos educativos de 2965 padres/ madres de niños escolarizados, pero a diferencia de esta investigación los niños no presentan problemas conductuales y se obtiene según un análisis factorial que con un resultado significativo los padres utilizan el factor inductivo o democrático al igual que está investigación, por lo que se establecen normas, pero se negocian y no se muestra conflicto en caso de cometer un error, sino que proveen apoyo a sus hijos a través de orientaciones; existe respeto y conocimiento mutuo, a la vez que se guía la actuación del hijo de forma racional. Son padres que se comunican frecuentemente y de forma abierta, donde el diálogo es el mejor sistema para la compresión.

De igual forma según un estudio realizado en Cajamarca- Perú por Cuenca y Marquina ${ }^{20}$ con una muestra de 264 estudiantes de diferentes instituciones educativas demuestran que tanto padres como madres obtuvieron ma- yores puntuaciones en el estilo autoritativo o inductivo, con lo que queda claro que en América Latina los estilos educativos o normas y reglas impuestas por los progenitores están basadas en ejercer autoridad, pero al mismo tiempo muestran preocupación, la cual se manifiesta por medio de la guía, fomentando la responsabilidad individual.

Cabe recalcar que el estudio tuvo diversas limitaciones como por ejemplo la influencia de padres en la respuesta de sus hijos, si bien es cierto esto no se dio de forma directa se pudo comprobar que los adolescentes contestaron a la escala en forma temerosa ya que sus padres podrían enterarse de sus respuestas, aunque se aclaró que la investigación es confidencial y por ninguna circunstancia se develarán nombres.

Por lo anterior, la autora del presente trabajo, recomienda realizar talleres en las instituciones educativas y centros vinculados a la atención integral de familias, que estén destinados a satisfacer las necesidades del sistema familiar, fortalezcan la relación y comunicación entre padres e hijos, preparen a padres para tener una mejor visión acerca de los diferentes estilos de crianza y se les enseñe sobre normas o exigencias adecuadas; ya que la conducta o comportamiento de los hijos y en este caso adolescentes se relacionan de manera significativa con las normas y reglas que son impuestas por los padres o cuidadores.

\section{Referencias Bibliográficas}

1. López S, Fernández A, Vives C, Rodríguez O. Prácticas de crianza y problemas de conducta en niños de educación infantil dentro de un marco intercultural. Redalyc. 2012;28:55-56.

2. Raya AF, Pino J, Herruzo J. La agresividad en la infancia: el estilo de crianza parental como factor relacionado. European Journal of Education and Psychology $\mathrm{N}^{\circ}$. 2009;2(3):213.

3. Torío López S, Peña Calvo JV, Inda Caro M. Estilos de educación familiar. Psicothema. 2008;20(1):62-70.

4. Izedin-Bouquet R, Pachajoa-Londoño A. Pautas , Prácticas Y Creencias Acerca De Crianza ... Ayer Y Hoy. Liberabit. 2009;15(2):109-115.

5. Valenzuela, M , Ibarra , A , Zubarew, T , \& Correa L. Prevención de conductas de riesgo en el Adolescente: Rol de familia. Scielo. 2013;22.

6. Borrás T. Adolescencia: definición, vulnerabilidad y oportunidad. scielo. 2014;18.

7. Limones I. ADOLESCENTES Y PERCEPCIÓN DEL SÍ MISMO: LA CONSTRUCCIÓN DE UNA IMAGEN REALISTA DE LA ADOLESCENCIA DESDE LA FAMILIA Y LA ESCUELA. Dialnet. 2011;11.

8. Zárate, Laura; Rivera, Elsa; González, María; León D. Problemas de conducta más frecuentes en jóvenes de secundaria. UNAM. 2009;12.

9. Martínez I, Fuentes MC, García F, Madrid I. estilo de socialización familiar como factor de prevención o riesgo para el consumo de sustancias y otros problemas 
de conducta en los adolescentes españoles. Adicciones. 17. Estupiñán E, Director M, Herrera Hernández JM. Trabajo 2013;25.

Fin De Grado Estilos Educativos En La Familia. 2014;.

10. Puello Scarpati M, Silva Pertuz M. Límites, reglas, comu- 18. Cuervo n. Pautas de crianza y desarrollo socioafectivo nicación en familia monoparental con hijos adolescentes. Diversitas: Perspectivas en Psicología. 2014;10(2):225246. en la infancia. Diversitas: Perspectivas en Psicología. 2009;6(1):111-121.

11. Garnica G, Robles R, Sánchez J, Juárez F. Mejoramiento de contingencias parentales y reducción de problemas conductuales en adolescentes. = Parental contingencies 20 . improvement and behavioral problems reduction in adolescents. Psicología Iberoamericana. 2013;21(1):79-87.

12. Ramírez M. PADRES Y DESARROLLO DE LOS HIJOS: PRACTICAS DE CRIANZA. Scielo. 2005;31.

13. Montañés M, Bartolomé R, Montañés J, Parra M. Influencia Del Contexto Familiar En Las Conductas Adolescentes. Ensayos. 2008;17:397.

14. Oliva Delgado A, Parra Jiménez A, Sánchez-Queija I, López Gaviño F. Estilos educativos materno y paterno: evaluación y relación con el ajuste adolescente. Anales de psicología. 2007;23(1):49.

19. Bersabé R, Fuentes MJ, Motrico E. Análisis psicométrico de dos escalas para evaluar estilos educativos parentales. Psicothema. 2001;13(4):678-684.

20. Cuenca L, Marquina Y. RELACIÓN ENTRE LOS ESTILOS DE CRIANZA Y EL BIENESTAR PSICOLÓGICO EN LOS ADOLESCENTES DE TERCERO Y CUARTO GRADO DE EDUCACIÓN SECUNDARIA DE LA CIUDAD DE CAJAMARCA _ Cuenca Torrel_RE. 2016;1.

15. Jiménez MJ. Estilos Educativos Parentales y su implicación en diferentes trastornos. 2010;p. 1-32.

16. Álvarez M. Prácticas educativas parentales : autoridad familiar, Parental educational practices : family effect on the children aggressive behavior authority, Pratiques éducatives parentales : autorité familiale, incidence dans la conduite agressive infantile. Revista Virtual Universidad Católica del Norte. 2010;Septiembre(31):253-273. 
